\title{
Service Loading Test as a Source for the Fatigue Curve Parameters' Estimation
}

\section{V. Gadolina and I. M. Petrova}

Blagonravov Institute of Engineering Science, Russian Academy of Sciences, Moscow, Russia

Very important question of fatigue curve parameter estimation might be explored from the point of view of service loading. Comparative testing at varied stress levels with subsequent computation might reveal some peculiarities of fatigue curves and substantial changes of their parameters due to service loading factors.

Keywords: metal fatigue, service loading, damage summation, nonlinear equation systems, test acceleration

Introduction. For the operation life estimation under random or block loading the fatigue curve (1) which describes the dependence of the ultimate number of cycles $N$ on the stress amplitude $\sigma$ by the varied kind of dependences (Wöhler, Weibull, et al.) is often used

$$
N=f(\sigma, \mathbf{A})
$$

where $\mathbf{A}$ is the fatigue curve parameters' vector which is usually estimated in the test with constant amplitude loading (one step loading).

Usage of the Mainer fatigue damage accumulation hypothesis

$$
\sum_{i=1}^{w} \frac{n_{i}}{N_{i}}=1
$$

for the total life estimation

$$
n_{\Sigma}=\sum_{i=1}^{w} n_{i}
$$

often leads to non-conservative estimation [1]. [In (3): $n_{i}$ is the number of the cycles with amplitude $\sigma_{i}$ and $w$ is the number of the intervals of the cyclecounted random loading process or the step number of the block loading.] That means that validity of (2) is not always approved experimentally. Numerous investigations show that the fatigue curve is affected by such loading factors as rare overloads, manifold loads below the endurance limit, etc.

The aim of the present work is to estimate the fatigue curve parameters with the utilization of the service loading test results. 
Assume we have the data of some service loading tests, which were carried out at $s$ loading levels. For reduction of errors due to the test data scatter inherent to fatigue, the assessment should include the statistical aspect. Usually 3 to 5 specimens are tested at each level. The value $n_{\Sigma}$ in (3) is a median value.

Let us define the calculated fatigue curve with the parameter vector $\mathbf{A}^{*}$ (A in general differs from $\mathbf{A})^{\oplus}$ as

$$
N^{*}=f_{1}\left(\sigma, \mathbf{A}^{*}\right)
$$

satisfying $s$ conditions of the Mainer linear summation (2):

$$
\begin{aligned}
& \sum_{i=1}^{w_{j}} \frac{n_{i, 1}}{N_{i, 1}^{*}\left(\sigma, \mathbf{A}^{*}\right)}=1, \\
& \sum_{i=1}^{w_{2}} \frac{n_{i, 2}}{N_{i, 2}^{*}\left(\sigma, \mathbf{A}^{*}\right)}=1, \\
& \ldots \\
& \ldots \\
& \sum_{i=1}^{w_{s}} \frac{n_{i, s}}{N_{i, s}^{*}\left(\sigma, \mathbf{A}^{*}\right)}=1 .
\end{aligned}
$$

Here $n_{1,1}, \ldots, n_{u_{1}, 1}$ are the components of the total life for the 1 -st test under service loading with stress amplitudes, respectively $\sigma_{1,1}, \ldots, \sigma_{w_{1}, 1}, n_{1, s}, \ldots, n_{w_{s}, s}$ are the same for the test number $s$ with stresses $\sigma_{1, s}, \ldots, \sigma_{w_{s} s}$. The number of the items $w_{k}$ in $k$ th test $(k=1,2, \ldots, s)$ corresponds to the number of intervals of the cycle-counted random loading processes or to the step number of the block loading.

The dimension $r$ of the parameter $\mathbf{A}^{*}$ depends on the kind of fatigue life described by Eq. (1) and is not lower than $2(r \geq 2)$. It defines the number of the required levels of testing $s(s \geq r)$.

Various approaches for the solution of the equation system (5) relative to unknown $\mathbf{A}^{*}$ were developed.

The Direct Selection of $\mathbf{A}^{*}$ Based on Consideration of Experimental Results of Comparative Irregular Testing. The approach is applied when only one parameter varies, for example, the fatigue limit $\sigma_{r}$.

For the structural steels under one-step loading often a threshold value (fatigue limit) is determined. In this case, Eq. (1) in nonlinear form and with additional parameter $\sigma_{r}$ has the form $(r=3)$ :

\footnotetext{
${ }^{(\mathbb{D}}$ Hereinafter symbol * depicts all values obtained by the comparative irregular testing computation method.
} 


$$
\begin{array}{lll}
\log N=C=m \log \sigma & \text { for } & \sigma>\sigma_{r}, \\
\log N=\infty & \text { for } & \sigma \leq \sigma_{r},
\end{array}
$$

where $C$ and $m$ are the material parameters.

Experiments have shown, that the fatigue limit $\sigma_{r}$ is often decreased due to operation factors. The design method for operation life estimation based on this phenomena has been developed [2]. This method assumes that parameter $\sigma_{r}$ decreases, and parameters $C$ and $m$ remain constant.

With the aim of experimental verification of the fatigue curve extrapolation in the upper part of the equation (6) below the fatigue limit $\sigma_{r}$, the irregular fatigue testing with two different loading blocks were carried out (two irregular experiments: $s=2$ ). In Fig. 1, those blocks are shown together with fatigue curves. Lines 1 show the initial 8-step loading block, in which two low steps are below the fatigue limit $\sigma_{r}$. Here $\sigma_{r}$ was estimated in constant loading test illustrated by fatigue curve 3 . Lines 2 depict the truncated block, in which two low steps were discarded. If only two low steps in block 1 do not contribute to the fatigue damage, the fatigue life expressed in the block numbers would remain the same. The distribution of fatigue lives expressed in block number significantly differs. This testifies to the fact, that low steps do contribute into the fatigue damage. The experimental results confirm that the fatigue limit decreases during the irregular loading, i.e., $\sigma_{r}^{*}<\sigma_{r}$, and fatigue curve changes from 3 to 4 .

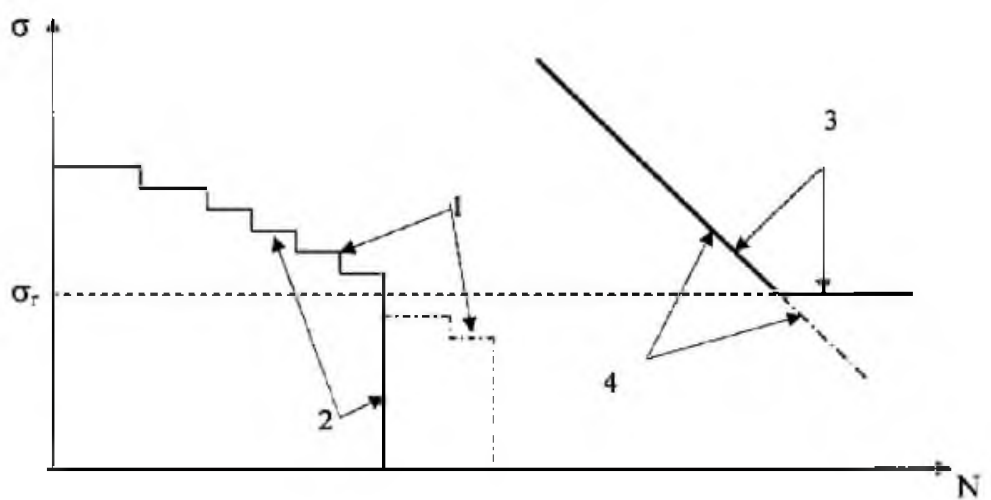

Fig. 1. Comparative tests under block loading.

Selection of $\mathbf{A}^{*}$ Based on Accelerated Coefficient Analysis $k_{a c}$. In [3], the approach of the acceleration coefficient $k_{a c}$ to the estimating fatigue curve parameters in gigacycle region $\left(N>10^{8}\right)$ was introduced. Value of $k_{a c}$ shows the ratio of the fatigue lives at varied loading levels. This approach is applied to homothetic stress blocks, i.e., for such tests, in which the non-dimensional distribution $\left(S_{i}, t_{i}\right)$ is constant $\left(S_{i}=\sigma_{i} / \sigma_{\max }, t_{i}=n_{i} / n_{\Sigma}, \sigma_{\max }\right.$ is the maximum amplitude in the loading block). The tests' number on varied levels should be $s>2$.

Let us consider the set of $G$ hypotheses based on the linear summation rule (2). Comparing the $k_{a c}$ values - experimental and calculated according to modified hypotheses - we can choose the best fit hypothesis (providing the best 
correlation). While doing this, the damage estimation is made using by the modified $G$ fatigue curves (the number of hypothesis corresponds to the number of fatigue curve). The selection of the most appropriate hypothesis is selected by the minimum value of $W^{(g)}$, where $\mathrm{g}$ is the order number of the hypothesis $(g=1,2, \ldots, G)$. By definition $k_{a c}>1$. If we have two levels of irregular testing ( $s=2$ ), then $k_{a c}=R_{\max } / R_{\min }$, where $R_{\max }$ and $R_{\min }$ are the median fatigue lives under lower and higher loading levels, respectively. Calculated according the hypothesis $g$, acceleration coefficients are $k_{a c}^{(g)}=R_{\max }^{(g)} / R_{\min }^{(g)}$, where $R_{\max }^{(g)}$ and $R_{\min }^{(g)}$ are the corresponding fatigue lives calculated by the gth hypothesis. criterion:

In this case, the following expression is proposed as an optimization

$$
W^{(g)}=\left|k_{y c}^{*}-k_{y c}^{(g)}\right| \rightarrow \min .
$$

For the analysis of test results with $s>2, s-1$ experimental and calculated acceleration coefficients are determined. For the selection of the optimum hypothesis the minimum of the additive value of the optimization criterion is chosen:

$$
W(g)=\sum_{i=1}^{s-1}\left|k_{a c, i}-k_{a c, i}^{(g)}\right| \rightarrow \min .
$$

Here

$$
k_{a c, i}=R_{i} / R_{\min } \quad \text { and } \quad k_{a c, i}^{(g)}=R_{i}^{(g)} / R_{\min }^{(g)},
$$

where $R_{i}$ and $R_{i}^{(g)}(i=1,2, \ldots, s-1)$ are respectively experimental and calculated according to gth hypothesis fatigue lives under lower stress levels.

The example of estimation of the modified fatigue curve slope $m^{*}$ is considered. Here the form of fatigue curve (1) was taken as

$$
\log N=C-m \log \sigma
$$

where $C$ and $m$ are the material parameters $(r=2)$.

The example of laboratory testing of the specimens under wide-band random loading at three levels $(s=3)$ was considered. Based on comparison of the calculated acceleration coefficients with experimentally obtained ones, the optimum value of $m^{*}=8-9$ was derived (the initial value, resulted from the constant amplitude test, reached $m=18$. In Fig. 2, the dependence of the additional optimization criterion (8) on varied slope parameter is shown. The first minimum of $W$ is for $m^{*}=8-9$. A dotted vertical line indicates $m$ value obtained in regular loading testing. The second minimum of the calculated dependence $W(m=17)==0.95$ is located nearby.

Non-Linear Equation System (5) Solution by the Least-Square Method. The approach is the most general. Superimposing unit to the left part, we convert the system (5) into the form: 


$$
\begin{aligned}
& \sum_{i=1}^{w_{1}} \frac{n_{i, 1}}{N_{i, 1}^{*}\left(\sigma, \mathbf{A}^{*}\right)}-1=0, \\
& \sum_{i=1}^{w_{2}} \frac{n_{i, 2}}{N_{i, 2}^{*}\left(\sigma, \mathbf{A}^{*}\right)}-1=0, \\
& \ldots \\
& \ldots \\
& \sum_{i=1}^{w_{s}} \frac{n_{i, s}}{N_{i, s}^{*}\left(\sigma, \mathbf{A}^{*}\right)}-1=0 .
\end{aligned}
$$

The solution of the system (5) will be the values of parameters $\mathbf{A}^{*}$, under which the deviations from zero (the objective function) of the Eqs. (10) are minimal.

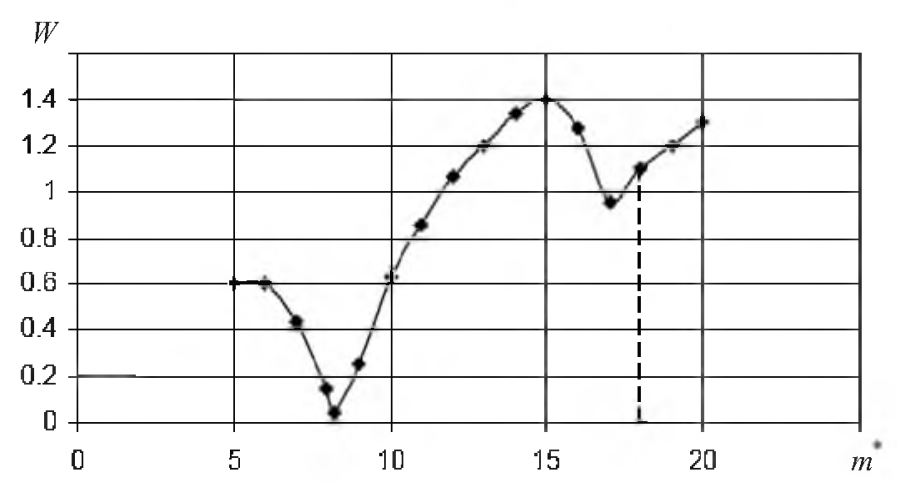

Fig. 2. Optimization additive criterion $W$ on slope parameter $m^{*}$ (modeling).

For the fatigue curve in form (6) the system (9) is converted to

$$
\begin{aligned}
& \sum_{i=1}^{m_{j}} \frac{n_{i, 1}}{10^{C-m \log \sigma_{i, 1}}}-1=0, \\
& \sum_{i=1}^{w_{2}} \frac{n_{i, 2}}{10^{C-m \log \sigma_{i, 2}}}-1=0, \\
& \ldots \\
& \ldots \\
& \sum_{i=1}^{w_{s}} \frac{n_{i, s}}{10^{C-m \lg \sigma_{i, s}}}-1=0 .
\end{aligned}
$$

Converting the expressions in denominator in (11) and denoting $10^{C}=C^{\prime}$, we get 


$$
\begin{aligned}
& \sum_{i=1}^{w_{1}} \frac{n_{i, 1}}{C^{\prime}} 10^{m \log \sigma_{i, 1}}-1=0, \\
& \sum_{i=1}^{w_{2}} \frac{n_{i, 2}}{C^{\prime}} 10^{m \log \sigma_{i, 2}}-1=0, \\
& \ldots \\
& \ldots \\
& \sum_{i=1}^{w_{s}} \frac{n_{i, s}}{C^{\prime}} 10^{m \log \sigma_{i, s}}-1=0 .
\end{aligned}
$$

The next step is the system (12) solution by the least square method. This method application is the most correct when the values have normal distribution If this condition is satisfied, the solution will correspond to the maximum likelihood method. If the distribution differs from normal, the problem should be solved by the maximum likelihood method taking into consideration the hypotheses of the real random value distribution.

To have fairly representative set by the irregular loading with two-parameter fatigue curve estimation (9) $(r=2)$, the number of tested levels $s$ (considering scatter) should be not less than $3(s \geq 3)$.

For the least square method system solution the standard algorithm in computation media MATLAB 6.5 was used. If the fatigue curve has only two parameters, the equation system solution might be obtained graphically, using computation mathematics graphical facilities.

If the fatigue curve is a step-wise non-linear function $(r>2)$, for example as in (6), the random adaptive search method should be used for its solution.

For the considered example of random loading, the calculated by the least square method result is almost the same as that obtained by $\mathbf{A}^{*}$ selection based on the accelerated coefficient analysis $k_{a c}$.

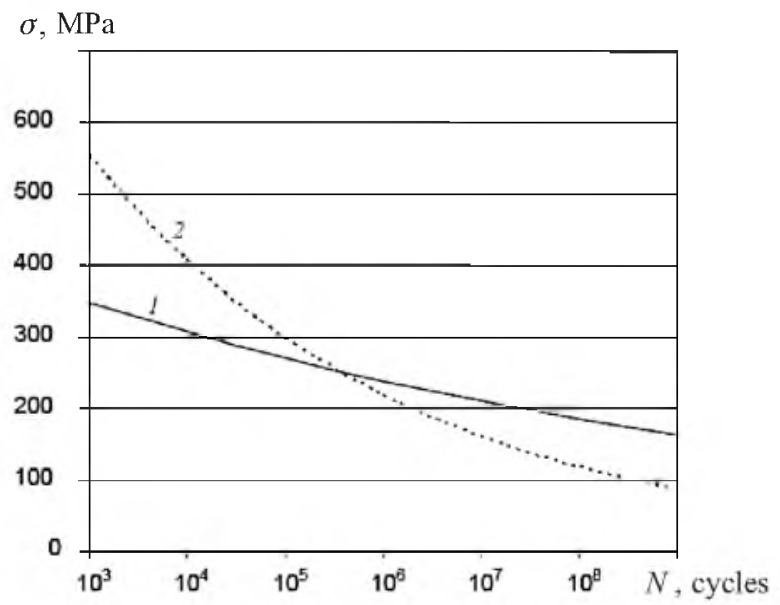

Fig. 3. Comparison of the experimental and calculated fatigue curves. 
In Fig. 3, the fatigue curve obtained for regular loading test is shown (solid line) together with the curve, obtained on the base of comparative random loading according to the proposed method.

Conclusions. Based on some examples of the block and random loading tests, the fatigue curve parameter $\mathbf{A}^{*}$ estimation by the proposed method has been performed. This estimation was obtained under conditions which were the

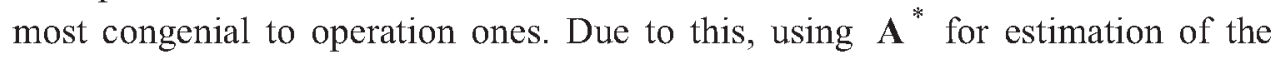
service life discounts the error probability. For the fatigue curve in form (6) it was found that the slope fatigue parameter in $\mathbf{A}^{*}$ is less fitting with $\mathbf{A}\left(m^{*}<m\right)$. This result agrees well with those obtained in [4].

1. A. P. Gusenkov (Ed.), V. P. Kogaev, Strength Calculations under Variable Stress Conditions [in Russian], Mashinostroenie, Moscow (1993).

2. A. P. Gusenkov (Ed.), I. M. Petrova, I. V. Gadolina, Fatigue Strength Analisis of Machines with Account of Probability Aspects [in Russian], Methodical Guideline: Problems of Strength, Operation Life, and Realiability of Machines, Moscow (1991).

3. I. V. Gadolina, "Tests under irregular loading as a source of information about the fatigue curve behavior in the gigacycle region," in: Mechanical Fatigue of Metals (Proc. of the Colloq.) [in Russian], Ternopil State University, Ternopil (2006), pp. 141-143.

4. M. E. Garf and E. D. Buglov, "On peculiarities of fatigue damage accumulation under a range of nonstationary loads below fatigue limit," Vest. Mashinostr., No. 6, 23-25 (1964). 